\title{
NOTIZEN
}

\section{Fieldgradient Two-Center Integrals by the Fourier Convolution Theorem}

Helmut Wrubel and Jürgen Voitländer

Sektion Physik und Physikalisch-Chemisches Institut der Universität München

(Z. Naturforsch. 24 a, 1660-1662 [1969] ; received 7 August 1969)

Using the Fourier convolution theorem GELLER ${ }^{1}$ has given analytical results for the two-center integrals $\left\langle\chi^{\mathrm{B}}\left|\hat{q}^{\mathrm{A}}\right| \chi^{\mathrm{B}}\right\rangle$, where $\chi^{\mathrm{B}}$ is a Slater orbital centered at nucleus $\mathrm{B}$ and $\hat{q}^{\mathrm{A}}$ is the operator of the electric field gradient centered at nucleus $\mathrm{A}$. There is another type - $\left\langle\chi^{\mathrm{A}}\left|\hat{q}^{\mathrm{A}}\right| \chi^{\mathrm{B}}\right\rangle$ - of two-center integrals for which one can obtain analytical results by means of the Fourier convolution theorem.

First the Fourier transforms of the product of the Slater orbital and the e.f.g. operator at nucleus A - $F\left(\boldsymbol{r}_{\mathrm{A}}\right)=\chi^{\mathrm{A}}(n, l, m) \cdot \hat{q}^{\mathrm{A}}-$ and of the Slater orbital centered at nucleus $\mathrm{B}-g\left(\boldsymbol{r}_{\mathrm{B}}\right)=\chi^{\mathrm{B}}(n, l, m)-$ have to be evaluated, then the product of these transforms has to be retransformed. Decomposing $F\left(\boldsymbol{r}_{\mathrm{A}}\right)$ according to different associated Legendre Polynomials $P_{L}^{M}\left(\cos \vartheta_{\mathrm{A}}\right)$ only expressions such as

$f\left(\boldsymbol{r}_{\mathrm{A}}\right)=$ const $r_{\mathrm{A}}^{N-4} \exp \left\{-\alpha r_{\mathrm{A}}\right\} P_{L}^{M}\left(\cos \vartheta_{\mathrm{A}}\right)\left\{\begin{array}{l}\cos M \varphi_{\mathrm{A}} \\ \sin M \varphi_{\mathrm{A}}\end{array}\right\}$

must be considered. For the calculation of the Fourier transforms the following expansion is adopted:

$$
\begin{aligned}
\exp \{ & \pm i \boldsymbol{k r}\} \\
= & \sum_{l=0}^{\infty}(2 l+1)( \pm i)^{l} j_{l}(k r) \sum_{m=0}^{l}\left(2-\delta_{m, 0}\right) \frac{(l-m) !}{(l+m) !} \\
& \times P_{l}^{m}(\cos \vartheta) P_{l}^{m}(\cos u) \cos (m(\varphi-v))
\end{aligned}
$$

where $k, u, v$ are the spherical coordinates of $\boldsymbol{k}$ and $j_{l}(k r)$ is the spherical Bessel function. With this expansion the angular integration can be carried out leaving only:

$$
\begin{gathered}
\tilde{f}(\boldsymbol{k})=\int f\left(\boldsymbol{r}_{\mathrm{A}}\right) \exp \left\{i \boldsymbol{k} \boldsymbol{r}_{\mathrm{A}}\right\} \mathrm{d} \tau \\
=\text { const } \int_{0}^{\infty} r_{\mathrm{A}}^{N-1} \exp \left\{-\alpha r_{\mathrm{A}}\right\} j_{\mathrm{L}}\left(k r_{\mathrm{A}}\right) \mathrm{d} r_{\mathrm{A}}, \\
\quad N=1,2,3, \ldots ; L=0,1,2, \ldots ; \\
\tilde{g}(\boldsymbol{k})=\int g\left(\boldsymbol{r}_{\mathrm{B}}\right) \exp \left\{i \boldsymbol{k} \boldsymbol{r}_{\mathrm{B}}\right\} \mathrm{d} \tau \\
=\text { const } \int_{0}^{\infty} r_{\mathrm{A}}^{N^{\prime}+1} \exp \left\{-\beta r_{\mathrm{B}}\right\} j_{L^{\prime}}\left(k r_{\mathrm{B}}\right) \mathrm{d} r_{\mathrm{B}}, \\
N^{\prime}=1,2, \ldots ; L^{\prime}=0,1, \ldots .
\end{gathered}
$$

Reprint requests to Doz. Dr. J. VoItländer, PhysikalischChemisches Institut der Universität München, D-8000 München 2, Sophienstr. 11.

1 M. Geller, J. Chem. Phys. 39, 84 [1963].
The remaining integrals are of the general form:

$$
G_{\mu, v}(\gamma, k)=\int_{0}^{\infty} r^{\mu} \exp \{-\gamma r\} j_{v}(k r) \mathrm{d} r .
$$

To treat these integrals $j_{l}(x)$ is expressed as ${ }^{2 a}$ (however not suitable for the case of $\mu=-1$; see below) :

$$
j_{l}(x)=\frac{1}{2 i^{l}} \int_{-1}^{+1} \exp \{i x t\} P_{l}(t) \mathrm{d} t .
$$

The integration can be interchanged. From the integration over $\mathrm{d} t$ a term involving $\log z$ can arise, which is transformed using the identity:

$$
\frac{1}{2 i} \log \frac{1+i z}{1-i z}=\operatorname{arctg} z .
$$

It is not necessary to evaluate all integrals (5). By means of partial integration of (5) and use of the recurrence formulae for $j_{l}(x)$ and its derivatives recurrence relations for the $G_{\mu, \nu}(\gamma, k)$ are obtained. As starting values only two of the integrals (5) need to be known.

If $\chi^{\mathrm{A}}$ describes a 1s-orbital [corresponding to $\mu=-1$ in (5)] noting

$$
\frac{\partial}{\partial \alpha} \frac{\exp \left\{-\alpha r_{\mathrm{A}}\right\}}{r_{\mathrm{A}}} j_{2}\left(k r_{\mathrm{A}}\right)=-\exp \left\{-\alpha r_{\mathrm{A}}\right\} j_{2}\left(k r_{\mathrm{A}}\right)
$$

the identity

$$
G_{-1,2}(\alpha, k)=-\int_{\infty}^{\alpha} G_{0,2}\left(\alpha^{\prime}, k\right) \mathrm{d} \alpha^{\prime}
$$

is obtained.

As an example the Fourier transform of a 2 s-orbital and $\hat{q}_{z z}$ is given:

$\tilde{f}(\boldsymbol{k})=$ const $\left(\left(\frac{1}{2 k}+\frac{3 a^{2}}{2 k^{3}}\right) \operatorname{arctg} \frac{k}{a}-\frac{3 a}{2 k^{2}}\right) P_{2}^{0}(\cos u)$.

Now to get the two-center integrals we must solve the inverse Fourier transforms.

$$
\begin{aligned}
I(\boldsymbol{R})= & (2 \pi)^{-3} \int_{0}^{\infty} \int_{0}^{\pi} \int_{0}^{2 \pi} \\
& \exp \{-i \boldsymbol{k} \boldsymbol{R}\} \tilde{f}(k) P_{L}^{M}(\cos u)\left\{\begin{array}{l}
\cos M v \\
\sin M v
\end{array}\right\} \\
& \cdot \tilde{g}(k) P_{L^{\prime}}^{M^{\prime}}(\cos u)\left\{\begin{array}{l}
\cos M^{\prime} v \\
\sin M^{\prime} v
\end{array}\right\} k^{2} \mathrm{~d} k \sin u \mathrm{~d} u \mathrm{~d} v .
\end{aligned}
$$

To evaluate these integrals expansion (2) is adopted but for $j_{l}(x)$ Rayleigh's formula is used $2 \mathrm{~b}$

2 M. Abramowitz and I. Stegun, Handbook of Mathematical Functions, Dover Publication, Inc., New York 1965, a) 10.1.14, b) 10.1.25. 


$$
j_{l}(x)=x^{l}\left(-\frac{1}{x} \frac{\mathrm{d}}{\mathrm{d} x}\right)^{l}\left(\frac{\sin x}{x}\right) .
$$

Integration of (10) over the angular part causes some of these integrals to vanish by symmetry. The integration over $\mathrm{d} k$ completes the analytical calculation. From Eq. (10) the remaining integrals are rewritten as $\left\langle k^{0} \mu \nu \gamma \delta l\right\rangle=\int_{0}^{\infty} G_{\mu, v}(\alpha, k) G_{\gamma, \delta}(\beta, k) j_{l}(k R) k^{2} \mathrm{~d} k$.

All necessary integrals are convergent. In order to evaluate these the Laplace transform

$$
\operatorname{arctg}(k / \alpha)=\int_{0}^{\infty} e^{-\alpha y} \frac{\sin (k y)}{y} \mathrm{~d} y
$$

is substituted. Integration over $\mathrm{d} k$ by means of the method of residues and then over $\mathrm{d} y$ (see appendix) is straightforward but rather tedious. The more complicated integrals are composed via recurrence relations from simpler ones. For example [using notation (12)] : $\frac{\mathrm{I}}{2}\left\langle k^{0} 00200\right\rangle-\frac{3}{2}\left\langle k^{-1} 01200\right\rangle=\left\langle k^{0} 02200\right\rangle$.

A rather great number of starting integrals $\left\langle k^{n} \mu \nu \gamma \delta l\right\rangle$ are necessary in order to obtain all integrals $\left\langle\chi^{\mathrm{A}}\left|\hat{q}^{\mathrm{A}}\right| \chi^{\mathrm{B}}\right\rangle$. The final result can be expressed by means of exponential integrals and related functions of the Slater exponents $\alpha, \beta$ and the two-center distance $R$.

For example the result for $\left\langle 1 \mathrm{~s}^{\mathrm{A}}\left|\hat{q}_{z z}^{\mathrm{A}}\right| 1 \mathrm{~s}^{\mathrm{B}}\right\rangle$ reads (including all normalizing factors) :

$$
\begin{aligned}
\left\langle 1 \mathrm{~s}^{\mathrm{A}}\left|\hat{q}_{z z}^{\mathrm{A}}\right|\right. & \left.1 \mathrm{~s}^{\mathrm{B}}\right\rangle=16 \alpha^{5 / 2} \beta^{5 / 2}\left\{\frac { e ^ { - \beta R } } { 1 2 \beta } \left[\frac{-2}{\alpha}+\frac{3 \alpha}{\beta^{2}}-\frac{6}{R \alpha \beta}+\frac{18 \alpha}{R \beta^{3}}-\frac{12}{R^{2} \beta^{2} \alpha}\right.\right. \\
+ & \left.\frac{45 \alpha}{R^{2} \beta^{4}}-\frac{12}{R^{3} \beta^{3} a}+\frac{45 \alpha}{R^{3} \beta^{5}}\right]+\left[-\alpha-\frac{5}{R}+\frac{4}{\alpha R^{2}}-\frac{15 \alpha}{R^{2} \beta^{2}}\right] \frac{e^{-\alpha R}}{4 R \beta^{4}} \\
+ & {\left[1-\frac{a^{2}}{\beta^{2}}+\frac{4}{R \beta}-\frac{6 \alpha^{2}}{R \beta^{3}}+\frac{9}{R^{2} \beta^{2}}-\frac{15 \alpha^{2}}{R^{2} \beta^{4}}+\frac{9}{R^{3} \beta^{3}}-\frac{15 a^{2}}{R^{3} \beta^{5}}\right] \cdot \frac{e^{-\beta R}}{8 \beta^{2}} 2 \int_{0}^{R} \frac{e^{-\alpha y}}{y} \sinh (\beta y) \mathrm{d} y } \\
+ & {\left.\left[\frac{\cosh (\beta R)}{4 \beta^{2}}\left(-1+\frac{\alpha^{2}}{\beta^{2}}-\frac{9}{R^{2} \beta^{2}}+\frac{15 \alpha^{2}}{R^{2} \beta^{4}}\right)+\left(4-\frac{6 \alpha^{2}}{\beta^{2}}+\frac{9}{R^{2} \beta^{2}}-\frac{15 \alpha^{2}}{R^{2} \beta^{4}}\right) \frac{\sinh (\beta R)}{4 R \beta^{2}}\right] E_{1}(R(\alpha+\beta))\right\} ; }
\end{aligned}
$$

The integral $2 \int_{0}^{R} \frac{e^{-\alpha y}}{y} \sinh (\beta y) \mathrm{d} y$ in (15) yields:

$$
2 \int_{0}^{R} \frac{e^{-\alpha y}}{y} \sinh (\beta y) \mathrm{d} y=\log \left|\frac{\alpha+\beta}{\alpha-\beta}\right|+E_{1}(R(\alpha+\beta))-E_{1}(R(\alpha-\beta)) .
$$

If $\alpha<\beta$ the principal value of the exponential integral is taken.

$$
\begin{gathered}
\text { Appendix } \\
I=\left\langle k^{-3} 0 \begin{array}{lll}
0 & 20 & 1
\end{array}\right\rangle=\frac{1}{R^{2}} \int_{0}^{\infty} \frac{\operatorname{arctg}(k / \alpha)[\sin (k R)-k R \cos (k R)]}{k^{4}\left(k^{2}+\beta^{2}\right)^{2}} \mathrm{~d} k .
\end{gathered}
$$

Making use of the Laplace transform (13), is the easiest way to treat such integrals. The sequence of integration may be interchanged. The integrand is an even function of $k$, therefore $I$ can be rewritten as $(u=y-R$, $x=y-R, w=y+R)$ :

$$
I=\frac{1}{4 R^{2}} \int_{0}^{\infty} \frac{e^{-\alpha y}}{y} \int_{-\infty}^{+\infty} \frac{\cos (x k)-\cos (w k)-[k R \sin (u k)+k R \sin (w k)]}{k^{4}\left(k^{2}+\beta^{2}\right)^{2}} \mathrm{~d} k \mathrm{~d} y .
$$

Application of the method of residues:

$$
\begin{aligned}
& I=\frac{1}{8 R^{2}} \int_{0}^{\infty} \frac{e^{-\alpha y}}{y}[\underbrace{\frac{e^{i x k}-e^{i w k}-(k R / i)\left(e^{i u k}+e^{i w k}\right)}{k^{4}\left(k^{2}+\beta^{2}\right)^{2}}}_{h_{1}(k)} \mathrm{d} k-\int_{h_{2}(k)}^{\int \frac{-e^{-i x k}+e^{-i w k}-(k R / i)\left(e^{-i u k}+e^{-i w k}\right)}{k^{4}\left(k^{2}+\beta^{2}\right)^{2}}} \mathrm{~d} k] \mathrm{d} y \\
& =\frac{1}{8 R^{2}} \int_{0}^{\infty} \frac{e^{-\alpha y}}{y} 2 \pi i\left(\left.\operatorname{Res} h_{1}(k)\right|_{i \beta}+\left.\operatorname{Res} h_{2}(k)\right|_{-i \beta}+\left.\operatorname{Res} h_{2}(k)\right|_{0}\right) \mathrm{d} y \\
& =\frac{-\pi}{8 R^{2} \beta^{7}}\left[( R \beta ^ { 2 } + \beta ) \frac { 2 e ^ { - \beta R } } { \beta ^ { 2 } - \alpha ^ { 2 } } \left(-\alpha+\alpha e^{-\alpha R} \cosh (\beta R)+\beta e^{-\alpha R} \sinh (\beta R) .\right.\right.
\end{aligned}
$$




$$
\begin{aligned}
& -2 e^{-\beta R}\left(R^{2} \beta^{2}+5 R+5\right) \int_{0}^{R} \frac{e^{-\alpha y}}{y} \sinh (\beta y) \mathrm{d} y+\frac{2}{3} \beta^{3}\left(\frac{2}{\alpha^{3}}-e^{-\alpha R}\left(\frac{R^{2}}{\alpha}+\frac{2 R}{\alpha^{2}}+\frac{2}{\alpha^{3}}\right)\right) \\
+ & \frac{1}{\alpha}\left(8 \beta-2 R^{2} \beta^{3}\right)\left(1-e^{-\alpha R}\right)+\left(2 R \beta^{2} \cosh (\beta R)-2 \beta \sinh (\beta R) \frac{e^{-(\alpha+\beta) R}}{\alpha+\beta}\right. \\
+ & 2\left(-R^{2} \beta^{2} \sinh (\beta R)+5 R \beta \cosh (\beta R)-5 \sinh (\beta R) E_{1}(R(\alpha+\beta))-\frac{4}{3} \beta^{3} R^{3} E_{1}(R \alpha)\right] .
\end{aligned}
$$

The authors are indebted to Mr. B. M. Ludwig for drawing their attention to a paper of KolKer and KARPLUs ${ }^{3}$. There these two-center integrals are evaluated by means of confocal elliptical coordinates. Using these coordinates some terms appear to diverge. Use of the Fourier convolution theorem avoids this difficulty.

3 H. J. Kolker and M. Karplus, J. Chem. Phys. 36, 960 [1962].

\section{Elektronen-Spin-Resonanz des $\mathrm{Cr}^{3^{3+}}$-Ions auf nichtkubischen Gitterplätzen in $\mathrm{SrTiO}_{3}$}

\section{Meierling}

II. Physikalisches Institut, Technische Hochschule, Darmstadt

(Z. Naturforsch. 24 a, 1662-1663 [1969] ; eingegangen am 1. August 1969)

ESR-spectra of $\mathrm{Cr}^{3+}$-ions on orthorhombic lattice sites in single crystals of $\mathrm{SrTiO}_{3}$ were observed at room temperature. The following constants were found:

$$
\begin{gathered}
g_{z}=1.9783 \pm 0,0005, \quad g_{x}=g_{y}=1.9761 \pm 0,0005, \\
|D|=(0.464 \pm 0.002) \mathrm{cm}^{-1}, \quad|E|=(0.272 \pm 0.005) \mathrm{cm}^{-1}, \\
|A|=(16.2 \pm 0.3) 10^{-4} \mathrm{~cm}^{-1} \text {. }
\end{gathered}
$$

Strontium-Titanat $\left(\mathrm{SrTiO}_{3}\right)$ gehört bei Temperaturen $T>110^{\circ} \mathrm{K}$ zur Raumgruppe $\mathrm{O}_{\mathrm{h}}{ }^{1}-\mathrm{Pm} 3 \mathrm{~m}$ (Perovskit). Im Zentrum der Elementarzelle befindet sich ein $\mathrm{Sr}^{2+}$-Ion. Die Würfelecken sind von $\mathrm{Ti}^{4+}$-Ionen besetzt, die ihrerseits oktaedrisch von $\mathrm{O}^{2-}$-Ionen umgeben sind. Die ESR-Spektren der $\mathrm{Fe}^{3+}$ - und $\mathrm{Cr}^{3+}$-Ionen im $\mathrm{SrTiO}_{3}$ wurden von MüLLER ${ }^{1}$ ausführlich untersucht. Er fand in beiden Fällen ein rein kubisches Spektrum. Später wurden weitere ESR-Untersuchungen am System $\mathrm{SrTiO}_{3}: \mathrm{Fe}^{3+}$ bekannt ${ }^{2,3}$; dabei fand man ein Spektrum, das charakteristisch ist für $\mathrm{Fe}^{3+}$-Ionen im Kristallfeld mit starker axialer Komponente. Für das axiale Kristallfeld macht man dabei eine $\mathrm{O}^{2-}$-Lücke verantwortlich, die sich in unmittelbarer Nachbarschaft des $\mathrm{Fe}^{3^{+}}$-Ions, das Ti-Plätze besetzt, befindet und damit zum Ladungsausgleich beiträgt.

Wir berichten hier über ein ESR-Spektrum von $\mathrm{Cr}^{3+}$. Ionen auf ortho-rhombischen Gitterplätzen in $\mathrm{SrTiO}_{3}$ bei Zimmertemperatur. Unsere Einkristalle waren mit 0,2 At.-Proz. $\mathrm{Cr}^{3+}$-Ionen dotiert (National Lead Comp. USA), sie absorbieren in $0,5 \mathrm{~mm}$ Schichtdicke auch nach mehrstündigem Tempern bei $1000{ }^{\circ} \mathrm{C}$ im $\mathrm{O}_{2}$-Strom den sichtbaren Spektralbereich vollständig.

Die ESR-Messungen wurden im X-Band (Varian 4502) und im Q-Band zunächst nur bei $T=293{ }^{\circ} \mathrm{K}$

Sonderdruckanforderungen erbeten an: Prof. Dr. B. ElschNER, II. Physikalisches Institut der Technischen Hochschule Darmstadt, D-6100 Darmstadt, Hochschulstr. 2.

1 K. A. Müller, Paramagnetic Resonance, Edit. W. Low, Acad. Press N. Y. 1963, Helv. Phys. Acta 31, 173 [1958]. durchgeführt. Neben dem starken kubischen Spektrum ${ }^{1}$ fanden wir ein Spektrum von $\mathrm{Cr}^{3+}$-Ionen auf orthorhombischen Gitterplätzen, dessen Absorptionslinien eine etwa 4000-mal kleinere Intensität haben als die starke isotrope $\mathrm{Cr}^{3+}$ Linie.

Bei X-Band-Messungen beobachtet man zwei Resonanzübergänge, wenn $H$ parallel zu einer vierzähligen Kristallachse angelegt wird. Ihre Halbwertsbreiten betragen 3,5 Oe für die Linie bei etwa $1,44 \mathrm{kOe}$ und 32 Oe für die Linie bei etwa 12,65 kOe (Abb. 1).

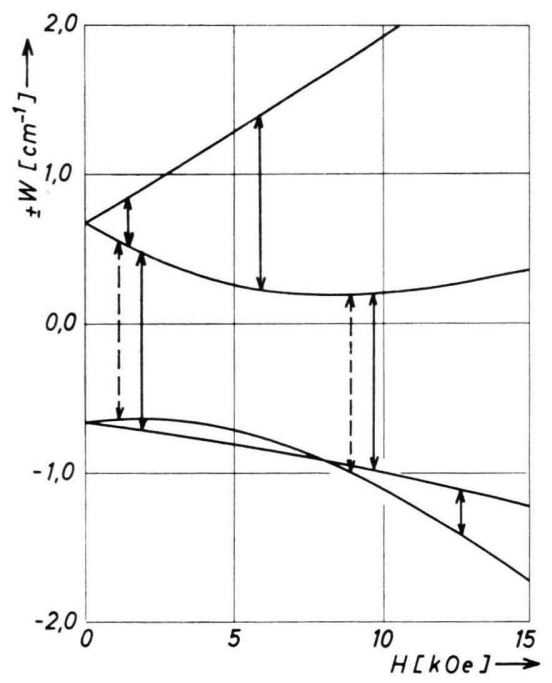

Abb. 1. Energieniveau-Schema für $H \|$ [100]. Beobachtete erlaubte Übergänge $(v=9464 \mathrm{MHz}$ und $35580 \mathrm{MHz})$ sind eingezeichnet. Gestrichelte Pfeile zeigen beobachtete „verbotene“ Übergänge an.

Das Spektrum ist mit dem folgenden Spin-HamiltonOperator $(S=3 / 2)$ zu beschreiben:

$\mathcal{H}=\beta \tilde{\boldsymbol{H}} \tilde{g} \boldsymbol{S}+D\left[S_{z}{ }^{2}-\frac{1}{3} S(S+1)\right]+E\left(S_{x}{ }^{2}-S_{y}{ }^{2}\right)+\boldsymbol{I} \tilde{A} \boldsymbol{S}$.

2 E. S. Kirkpatrik, K. A. Müller u. R. S. Rubins, Phys. Rev. 135, A 86 [1964].

3 R. Baer, G. Wessel u. R. S. Rubins, J. Appl. Phys. 39, 23 [1968]. 